\title{
Preface
}

\section{Recent advances in wide hybridization and embryo rescue of floricultural plants in Japan}

\author{
Masaru Nakano ${ }^{1}$ and Masahiro $\mathrm{Mii}^{2}$ \\ ${ }^{1}$ Faculty of Agriculture, Niigata University; ${ }^{2}$ Graduate School of Horticulture, Chiba University
}

In floricultural plants, the main breeding objective is to increase genetic variability in ornamental traits such as flower color, flower shape and plant form. To achieve this objective, cross breeding and mutation breeding are well established strategies for diverse floricultural plants. More recently, molecular breeding by genetic transformation is beginning to be applied to floricultural plants. Among these various breeding methods, wide hybridization, i.e., interspecific or intergeneric hybridization, may be most effective for increasing genetic variation.

Wide hybridization is suitable for practical breeding in floriculture for two reasons. First, most crop plants are propagated by seeds, in which repeated selfing or backcrossing of hybrids is required to fix the traits. However, interspecific and intergeneric hybrids generally show male and/or female sterility, and thus the hybrids cannot be used directly for subsequent breeding. Most floricultural plants, on the other hand, are propagated vegetatively, and sterile hybrids can be used directly as novel cultivars. Second, interspecific and intergeneric hybrids generally show an intermediate morphology between the two parents, which is usually undesirable for crop plants but is often attractive in floriculture, where novelty in ornamental traits is important for the development of cultivars.

In wide hybridization, however, cross-incompatibility barriers often inhibit the development of progeny plants. Embryo rescue techniques such as embryo, ovule and ovary cultures can partly overcome such barriers, and many interspecific or intergeneric hybrids have successfully been obtained by embryo rescue in a wide range of plant species. In floricultural plants, numerous hybrid cultivars have already been produced, especially in lilies and orchids, and wide hybridization has recently been applied to the breeding of various floricultural plants in other taxa.

This issue of Plant Biotechnology presents recent successful results on wide hybridization of floricultural plants in Japan. These include the production of interspecific hybrids in Primula, Primulaceae (Kato et al. pp 521-528), Cyclamen, Primulaceae (Ishizaka, pp 511-519) and Hydrangea, Hydrangeaceae (Kudo et al. pp 529-533), and of intergeneric hybrids in Asteraceae (Ohtsuka and Inaba, pp 535-539). We hope that the information in these feature articles will be valuable not only to breeders and researchers in floriculture but also to those working with crop plants.

Finally, we would like to express our deep gratitude to Prof. Masaaki Umeda, the editor-in-chief of Plant Biotechnology, for his continuous help in preparing this issue, and also to Ms. Kuniko Yasumi, the manuscript manager of Plant Biotechnology, for her kind and efficient assistance.

The editing of this special issue was supported by the invited editors, Dr. Takashi Handa (Meiji University) and Dr. Ryo Ohsawa (University of Tsukuba). The chief editor deeply appreciates their assistance. 